\title{
Retrospektive Analyse zur Dosisintensität von Epirubicin beim Mammakarzinom
}

\author{
G. Konecny M. Frank S. Kahlert M. Untch H. Hepp \\ Klinik und Poliklinik für Frauenheilkunde und Geburtshilfe im Klinikum Grosshadern der \\ Ludwig-Maximilians-Universität München, Deutschland
}

\section{Schlüsselwörter}

Mammakarzinom $\cdot$ Chemotherapie $\cdot$ Epirubicin, Dosierung

\section{Zusammenfassung}

Fragestellung: Die Wirksamkeit von Epirubicin wird in der Therapie des Mammakarzinoms möglicherweise von der applizierten Dosisintensität (DI) beeinflusst. Wir haben den Einfluss der tatsächlich applizierten DI auf die Verträglichkeit und das Ansprechen in der adjuvanten und metastasierten Situation überprüft. Patientinnen und Methoden: Epirubicin, Fluorouracil und Cyclophosphamid (FEC) wurden entweder in einer Dosierung von $500 / 50 / 500 \mathrm{mg} / \mathrm{m}^{2}$ $\left(\mathrm{FE}_{50} \mathrm{C}\right)$ oder in einer intensivierten Dosierung von $500 / 75 / 500 \mathrm{mg} / \mathrm{m}^{2}$ $\left(\mathrm{FE}_{75} \mathrm{C}\right)$ 3-wöchentlich gegeben. Die tatsächlich applizierte DI wurde berechnet und mit den Nebenwirkungen und dem Therapieansprechen korreliert. Von 66 Patientinnen waren 63 auswertbar; 43 hatten eine metastasierte Erkrankung, und 20 wurden adjuvant behandelt. Ergebnisse: Adjuvant wurden für $\mathrm{FE}_{50} \mathrm{C}$ und $\mathrm{FE}_{75} \mathrm{C} 81$ bzw. 96\% der geplanten DI und 70 bzw. $88 \%$ der geplanten Gesamtdosis tatsächlich appliziert. In der metastasierten Situation wurden 94 bzw. 92\% der geplanten $\mathrm{FE}_{50} \mathrm{C}$ - bzw. $\mathrm{FE}_{75} \mathrm{C}$-DI gegeben. Dies entsprach einer tatsächlichen Erhöhung der DI von Epirubicin um $81 \%$ in der adjuvanten und um $53 \%$ in der metastasierten Situation. Die hämatologischen und nicht hämatologischen Toxizitäten beider Regime waren vergleichbar. Im retrospektiven Vergleich liess sich das mittlere Gesamtüberleben nach 4 Jahren in der adjuvanten Situation von 40 auf $48 \%$ verbessern $(p=0.47)$, und die Remissionsrate beim metastasierten Mammakarzinom konnte von 34 auf $44 \%$ erhöht werden. Schlussfolgerung: Die applizierte DI von Epirubicin war in allen Gruppen niedriger als initial geplant. Höhere DI von Epirubicin führten beim primären und fortgeschrittenen Mammakarzinom zu vergleichbaren Toxizitäten. Die Einhaltung der geplanten DI scheint eine wichtige Voraussetzung für den optimalen Therapieerfolg zu sein. Unsere Ergebnisse unterstreichen ferner die Notwendigkeit, eine Intensivierung von Anthrazyklinen beim Mammakarzinom weiter in prospektiven Studien zu untersuchen.

\author{
Key Words \\ Breast cancer - Chemotherapy - Epirubicin, dosage
}

\section{Retrospective Analysis of Dose Intensity of Epirubicin in Breast Cancer}

Objective: In breast cancer the efficacy of epirubicin-based chemotherapy is possibly related to the actual dose intensity applied. We retrospectively determined the administered dose intensity and the relative toxicity and efficacy of an epirubicin-containing regimen in patients with primary or metastatic breast cancer. Patients and Methods: Fluorouracil, epirubicin, and cyclophosphamide (FEC) were either given at a standard dose of $500 / 50 / 500 \mathrm{mg} / \mathrm{m}^{2}\left(\mathrm{FE}_{50} \mathrm{C}\right)$ or at an intensified dose of $500 / 75 / 500 \mathrm{mg} / \mathrm{m}^{2}\left(\mathrm{FE}_{75} \mathrm{C}\right)$ every 3 weeks. Of the 66 patients treated, 63 were evaluable; 43 had metastatic breast cancer, and 20 patients with an increased risk of relapse received FEC as an adjuvant treatment. Results: Dose intensity and absolute dose of adjuvant treatment were 81 and $70 \%$ for $\mathrm{FE}_{50} \mathrm{C}$ and 96 and $88 \%$ for $\mathrm{FE}_{75} \mathrm{C}$. In metastatic breast cancer, the dose intensity for $\mathrm{FE}_{50} \mathrm{C}$ was $94 \%$ and for $\mathrm{FE}_{75} \mathrm{C} 92 \%$. In a retrospective comparison, the 4-year overall survival following adjuvant $\mathrm{FE}_{50} \mathrm{C}$ and $\mathrm{FE}_{75} \mathrm{C}$ was 40 and $48 \%$, respectively $(p=0.47)$. The dose intensification led to a higher response rate of $34 \mathrm{vs.} 44 \%$. There were no significant differences in response duration and survival time. The toxicity profiles were comparable between $\mathrm{FE}_{50} \mathrm{C}$ and $\mathrm{FE}_{75} \mathrm{C}$. Conclusions: In general, the doses applied were lower than initially planned. Higher doses of epirubicin did not result in a significant increase of toxicity. Despite the limitations of a retrospective analysis, our observations support the importance of adherence to the planned dose intensity as a prerequisite for optimal treatment of patients suffering from breast cancer. As our results could be related to selection bias, dose-intensified anthracycline-containing regimens should be further evaluated in prospective trials.

Copyright $\odot 2001$ S. Karger AG, Basel

\begin{tabular}{ll}
\hline KARGER & ○ 2001 S. Karger AG, Basel \\
Fax +4161306 12 34 & \\
$\begin{array}{l}\text { E-Mail karger@karger.ch } \\
\text { www.karger.com }\end{array}$ & $\begin{array}{l}\text { Accessible online at: } \\
\text { www.karger.com/journals/ggr }\end{array}$
\end{tabular}

\footnotetext{
Gottfried Konecny, UCLA School of Medicine, Dept. of Medicine Division of Hematology-Oncology, 12-145 Factor Building Los Angeles, CA 90095 (USA), Tel. +1 3102064156 Fax +1 310825 6192, E-Mail gkonecny@ucla.edu 


\section{Analyse rétrospective sur l'intensité de la dose d'épirubicine dans le cancer du sein}

Objectif: L'efficacité de l'épirubicine dans le traitement du cancer du sein dépend vraisemblablement de l'intensité de la dose (ID) effectivement administrée. Nous en avons étudié l'influence sur la tolérance et la réponse. Le traitement était appliqué, soint en tant que traitement adjuvant, soit pour des métastases. Patientes et méthodes: L'épirubicine, le fluorouracile et le cyclophosphamide (FEC) ont été administrés à intervalles de 3 mois à la dose prévue de 500/50/500 $\mathrm{mg} / \mathrm{m}^{2}\left(\mathrm{FE}_{50} \mathrm{C}\right)$ ou à une posologie plus forte de $500 / 75 / 500 \mathrm{mg} / \mathrm{m}^{2}$ $\left(F E_{75} C\right)$. L'ID effectivement administrée a été calculée et corrélée aux effets secondaires et à la résponse thérapeutique. Soixante-trois patientes sur les 66 ont pu être évaluées: 43 étaient porteuses de métastases et $\mathbf{2 0}$ ont bénéficié d'un traitement adjuvant. Résultats: En ce qui concerne le traitement adjuvant, 81 et $96 \%$ de I'ID de $\mathrm{FE}_{50} \mathrm{C}$ et $\mathrm{FE}_{75} \mathrm{C}$ et 70 et $88 \%$, de la dose totale prévue ont été effectivement administrés. Dans le cas de métastases, 94 et $92 \%$ de I'ID ont été administrés pour $\mathrm{FE}_{50} \mathrm{C}$ et de $\mathrm{FE}_{75} \mathrm{C}$. Ceci correspond à une augmentation de I'ID d'épirubicine de $81 \%$ pour le traitement adjuvant et de $53 \%$ pour le traitement de métastases. Les toxicités hématologique et non hématologique des deux traitements ont été comparables. L'analyse rétrospective a fait apparaître une amélioration de la survie moyenne à 4 ans de $40 \%$ pour le traitement adjuvant et de $48 \%$ pour le traitement des métastases $(p=0.47)$ et le taux de rémission chez les patientes porteuses de métastases de cancer du sein a passé de 34 à 44\%. Conclusions: Dans les deux groupes, I'ID d'épirubicine a été plus faible que prévu. Des ID d'épirubicine augmentées ont eu une toxicité comparable dans les deux groupes. Le respect de I'ID prévue semble constituer une condition indispensable au succès thérapeutique. Nos résultats soulignent par ailleurs la nécessité de conduire des études prospectives sur l'opportunité d'intensifier la dose d'anthracyclines dans le cancer du sein.

\section{Einleitung}

Die Anthrazykline Doxorubicin und Epirubicin zählen zu den aktivsten Substanzen für die systemische Behandlung des Mammakarzinoms. In der adjuvanten Therapie werden derzeit bei einem erhöhten Rezidivrisiko mit $>3$ positiven axillären Lymphknoten anthrazyklinhaltige Chemotherapieprotokolle empfohlen, da die Hinzunahme eines Anthrazyklins in mehreren Studien zu einem verbesserten rezidivfreien Überleben sowie zu einem verbesserten Gesamtüberleben geführt hat (Tab. 1) [1-8]. In der metastasierten Situation zeichnen sich anthrazyklinhaltige Schemata wie FEC, FAC, EC und AC im Vergleich zu CMF durch eine höhere Wirksamkeit aus, die sich in höheren Remissionsraten und in einem rascheren Wirkungseintritt widerspiegelt, wobei jedoch bisher nur in zwei randomisierten Studien eine signifikante Verbesserung des Gesamtüberlebens belegt werden konnte (Tab. 2) [9-13]. Einige retrospektive [14, 15] und prospektive [16, 17] Studien geben Hinweise darauf, dass eine Dosisintensivierung von Anthrazyklinen in der adjuvaten Therapie zu einer Verbesserung des rezidivfreien Überlebens und teilweise des Gesamtüberlebens führen kann. Beim metastasierten Mammakarzinom führte die
Dosisintensivierung in einigen Studien ebenso zu höheren Remissionsraten, wobei bisher aber kein Überlebensvorteil nachgewiesen werden konnte [18-21]. Wir haben daher den Einfluss einer Dosisintensivierung von Epirubicin an einem eigenen Patientenkollektiv der Frauenklinik im Klinikum Grosshadern in der adjuvanten und metastasierten Situation untersucht.

\section{Patientinnen und Methoden}

Zwischen Mai 1993 und Februar 1995 erhielten 66 Mammakarzinompatientinnen an der Frauenklinik im Klinikum Grosshadern sechs Zyklen FEC-Chemotherapie 3-wöchentlich. Im ersten Studienzeitraum erhielten Patientinnen FEC in einer Standarddosierung von $500 / 50 / 500 \mathrm{mg} / \mathrm{m}^{2}\left(\mathrm{FE}_{50} \mathrm{C}\right)$. Im weiteren Studienverlauf wurde die geplante Epirubicindosis von 50 auf $75 \mathrm{mg} / \mathrm{m}^{2}$ erhöht, FEC wurde dann dosisintensiviert mit 500/75/500 mg/m² $\left(\mathrm{FE}_{75} \mathrm{C}\right)$ appliziert. Von 63 auswertbaren Patientinnen wurden 20 adjuvant bzw. neoadjuvant behandelt. Für diese Patientinnen betrug die mittlere Followup-Periode 32 Monate. Weitere 43 Patientinnen hatten eine metastasierte Erkrankung, für die die mittlere Follow-up-Periode 15 Monate betrug. Von diesen Patientinnen erhielten 39 FEC als First-line- und 4 als Second-line-Therapie. Die Stadieneinteilung erfolgte nach der TNM-Klassifikation. Die Definition des Menopausenstatus erfolgte nach Anamnese oder laborchemischer Bestimmung der $\mathrm{E}_{2} / \mathrm{FSH}-$ Werte. Der Hormonrezeptorstatus wurde quantitativ mit einem Enzymimmunoassay bestimmt (Cutoff $10 \mathrm{fmol} / \mathrm{mg}$ Protein). Rezidivfreies Überleben, Gesamtüberleben und Remissionsdauer wurden nach Kaplan und Meier [22] berechnet. Die Signifikanzberechnungen in den univariaten Analysen wurden nach dem Log-rankVerfahren durchgeführt. In der adjuvanten Therapie erfolgte die Indikation zur anthrazyklinhaltigen Chemotherapie auf Grund eines erhöhten Rezidivrisikos ( $>3$ positive axilläre Lymphknoten, grosser Primärtumor, Haut- oder Pektoralisinfiltration oder Vorliegen eines inflammatorischen Karzinoms). In der metastasierten Situation erfolgte die Indikation zur anthrazyklinhaltigen Therapie bei überwiegend viszeraler Metastasierung oder starker Beschwerdesymptomatik. Hämatopoetische Wachstumsfaktoren wurden nicht verabreicht. Zur antiemetischen Therapie wurden Metoclopramid und, nach der Einführung der Serotoninantagonisten, Ondansetron $2 \times 8 \mathrm{mg} /$ Tag während 2-3 Tagen gegeben. Alle Patientinnen wurden eingehend über die Risiken und den zu erwartenden Nutzen der anthrazyklinhaltigen Chemotherapie aufgeklärt. Gemeinsam mit den Patientinnen wurden Vorgehensweise und Art des Therapieschemas ausgewählt.

\section{Ergebnisse}

Die Patientencharakteristika sind in den Tabellen 3 und 4 dargestellt. Bei einer 3-wöchentlichen Applikation von $\mathrm{FE}_{50} \mathrm{C}$ bzw. $\mathrm{FE}_{75} \mathrm{C}$ ergibt sich eine geplante Dosisintensität (DI) für Epirubicin von 16 bzw. $25 \mathrm{mg} / \mathrm{m}^{2 /}$ Woche. Im adjuvant behandelten Kollektiv wurde dagegen nur eine DI für Epirubicin von 13 bzw. $24 \mathrm{mg} / \mathrm{m}^{2} /$ Woche nach $\mathrm{FE}_{50} \mathrm{C}$ oder $\mathrm{FE}_{75} \mathrm{C}$ erreicht, was 81 bzw. 96\% der geplanten DI entsprach. Aufgrund der Diskrepanz zwischen geplanter und applizierter DI war nicht, wie geplant, eine Erhöhung der DI um 50\%, sondern tatsäch- 
Tabelle 1. Übersicht prospektiv randomisierter Studien zum Stellenwert der Anthrazykline in der adjuvanten Therapie des Mammakarzinoms

\begin{tabular}{|c|c|c|c|c|c|}
\hline Studie & $\mathrm{n}$ & Regime & $\begin{array}{l}\text { Follow-up- } \\
\text { Monate }\end{array}$ & $\begin{array}{l}\text { Rezidivfreies } \\
\text { Überleben, \% }\end{array}$ & $\begin{array}{l}\text { Gesamt- } \\
\text { überleben, } \%\end{array}$ \\
\hline $\begin{array}{l}\text { Oncofrance } \\
\mathrm{N}+[1]\end{array}$ & 249 & $\begin{array}{l}\mathrm{AVCF} \\
\mathrm{CMF}\end{array}$ & 192 & $\begin{array}{l}53 * \\
36\end{array}$ & $\begin{array}{l}56^{*} \\
41\end{array}$ \\
\hline $\begin{array}{l}\text { NSABP B-11 } \\
\mathrm{N}+, \text { ER- }[2]\end{array}$ & 697 & $\begin{array}{l}\text { PAF } \\
\text { PF }\end{array}$ & 72 & $\begin{array}{l}51^{*} \\
44\end{array}$ & $\begin{array}{l}65^{*} \\
59\end{array}$ \\
\hline $\begin{array}{l}\text { NSABP B-12 } \\
\mathrm{N}+, \mathrm{ER}+[2]\end{array}$ & 1093 & $\begin{array}{l}\text { PAFT } \\
\text { PFT }\end{array}$ & 72 & $\begin{array}{l}64 \\
63\end{array}$ & $\begin{array}{l}77 \\
78\end{array}$ \\
\hline $\begin{array}{l}\text { ICCG } \\
<50, N+[3]\end{array}$ & 636 & $\begin{array}{l}\text { FEC } \\
\text { CMF }\end{array}$ & 54 & $\begin{array}{l}85 * \\
82\end{array}$ & $\begin{array}{l}93 * \\
89\end{array}$ \\
\hline $\begin{array}{l}\text { SWOG } 8313 \\
\text { N+, ER- }[4]\end{array}$ & 331 & $\begin{array}{l}\text { FAMC } \\
\text { CMFVP }\end{array}$ & 60 & $\begin{array}{l}50 \\
55\end{array}$ & $\begin{array}{l}61 \\
64\end{array}$ \\
\hline $\begin{array}{l}\text { NSABP B-15 } \\
\mathrm{N}+,<50 \text { oder } \\
<60, \text { PR- }[5]\end{array}$ & 2194 & $\begin{array}{l}\text { AC } \\
\text { CMF } \\
\text { AC-CMF }\end{array}$ & 36 & $\begin{array}{l}62 \\
63 \\
68\end{array}$ & $\begin{array}{l}83 \\
82 \\
81\end{array}$ \\
\hline $\begin{array}{l}\text { Swedish Trial } \\
<50, N_{0} G_{2-3} \text { oder } \\
N+\text { ER- }[6]\end{array}$ & 874 & $\begin{array}{l}\text { FEC } \\
\text { CMF }\end{array}$ & 72 & & $\begin{array}{l}76^{*} \\
69\end{array}$ \\
\hline $\begin{array}{l}\text { NCI Canada } \\
<50, N+[7]\end{array}$ & 710 & $\begin{array}{l}\text { FEC } \\
\text { CMF }\end{array}$ & 60 & $\begin{array}{l}63 * \\
53\end{array}$ & $\begin{array}{l}77 * \\
70\end{array}$ \\
\hline $\begin{array}{l}\text { Southeastern Cancer } \\
\text { Study Group } \\
\mathrm{N}+[8]\end{array}$ & 528 & $\begin{array}{l}\text { FAC } \\
\text { CMF }\end{array}$ & 72 & & $\begin{array}{l}74 \\
68\end{array}$ \\
\hline
\end{tabular}

$\mathrm{N}+=$ Nodal positiv $; \mathrm{N}_{0}=$ nodal negativ; $\mathrm{ER}-=$ Östrogenrezeptor negativ; $\mathrm{ER}+=$ Östrogenrezeptor positiv; $\mathrm{PR}-=$ Progesteronrezeptor negativ; $<50=$ prämenopausal; $\mathrm{G}_{2-3}=$ Grading $2-3 ; \mathrm{A}=$ Doxorubicin; $\mathrm{V}=\mathrm{Vincristin} ; \mathrm{C}=$ Cyclophosphamid; $\mathrm{F}=$ Fluorouracil; $\mathrm{M}=$ Methotrexat; $\mathrm{P}=$ Prednisolon; $\mathrm{E}=$ Epirubicin; $\mathrm{T}=$ Tamoxifen .

* Signifikanter Unterschied.

Tabelle 2. Prospektiv randomisierte Studien zum Stellenwert von Anthrazyklinen in der Therapie des fortgeschrittenen Mammakarzinoms

\begin{tabular}{|c|c|c|c|c|}
\hline Regime & $\mathrm{n}$ & $\begin{array}{l}\text { Remissionsrate } \\
\%\end{array}$ & $\begin{array}{l}\text { Gesamtüberleben } \\
\text { Monate }\end{array}$ & Literatur \\
\hline CAFVP vs. CMFVP & 216 & 71 vs. 50 & 19 vs. $13^{*}$ & 9 \\
\hline CAF vs. CMF & 181 & 55 vs. 37 & 24 vs. $18^{*}$ & 10 \\
\hline CAF vs. CMF & 78 & 82 vs. 62 & 27,2 vs. 17 & 11 \\
\hline CAF vs. CMFP & 155 & 53 vs. 53 & 18,6 vs. 15,8 & 12 \\
\hline CAF vs. CMFVP & 265 & 55 vs. 40 & 22 vs. 19 & 13 \\
\hline
\end{tabular}

lich eine Erhöhung um $81 \%$ erfolgt. Adjuvant wurden nach $\mathrm{FE}_{50} \mathrm{C}$ und $\mathrm{FE}_{75} \mathrm{C}$ im Mittel $357 \mathrm{mg}$ und $671 \mathrm{mg}$ Epirubicin appliziert, was 70 bzw. 88\% der geplanten Gesamtdosis entsprach. Nach adjuvanter $\mathrm{FE}_{50} \mathrm{C}$ - oder $\mathrm{FE}_{75} \mathrm{C}$-Therapie betrug das mittlere rezidivfreie Überleben nach 4 Jahren 20 bzw. 40\% und das Gesamtüberleben 40 bzw. $48 \%$ (Abb. 1). Das mittlere rezidivfreie Intervall betrug nach $\mathrm{FE}_{50} \mathrm{C} 20$ Monate und nach $\mathrm{FE}_{75} \mathrm{C} 33$
Monate $(p=0,2)$ und die mittlere Gesamtüberlebenszeit 32 bzw. 39 Monate $(p=0,47)$.

In der metastasierten Situation wurde nach $\mathrm{FE}_{50} \mathrm{C}$ und $\mathrm{FE}_{75} \mathrm{C}$ eine DI Epirubicin von 15 bzw. $23 \mathrm{mg} / \mathrm{m}^{2} /$ Woche erreicht, was 94 und $92 \%$ der geplanten DI entsprach. Als absolute Dosen wurden in der metastasierten Situation im Mittel 403 bzw. 714 mg Epirubicin appliziert. Im Mittel wurden 4,5 Zyklen $\mathrm{FE}_{50} \mathrm{C}$ und 5,7 Zyklen $\mathrm{FE}_{75} \mathrm{C}$ ver- 
Tabelle 3. Charakteristika der adjuvant therapierten Patientinnen

\begin{tabular}{|c|c|c|}
\hline & $\mathrm{FE}_{75} \mathrm{C}$ & $\mathrm{FE}_{50} \mathrm{C}$ \\
\hline Auswertbare Patientinnen & 14 & 6 \\
\hline Mittleres Alter, Jahre (Bereich) & $46(30-65)$ & $58(40-74)$ \\
\hline \multicolumn{3}{|l|}{ Histologie } \\
\hline Invasiv-duktal & 10 & 3 \\
\hline Lobulär & 1 & 0 \\
\hline Inflammatorisch & 3 & 3 \\
\hline \multicolumn{3}{|l|}{ Operation } \\
\hline Tumorentfernung & 4 & 1 \\
\hline Erweiterte Mastektomie & 10 & 5 \\
\hline \multicolumn{3}{|l|}{ Positive Lymphknoten } \\
\hline $1-3$ & 8 & 1 \\
\hline$>3$ & 6 & 5 \\
\hline Im Mittel befallene Lymphknoten & 10 & 8 \\
\hline \multicolumn{3}{|l|}{ Hormonrezeptorstatus } \\
\hline Positiv ${ }^{\mathrm{a}}$ & 7 & 3 \\
\hline Negativ & 7 & 3 \\
\hline Prämenopausal & 12 & 2 \\
\hline Postmenopausal & 2 & 4 \\
\hline \multicolumn{3}{|l|}{ Tumorgrösse } \\
\hline $\mathrm{T}_{1}$ & 0 & 1 \\
\hline $\mathrm{T}_{2}$ & 5 & 0 \\
\hline $\mathrm{T}_{3}$ & 3 & 0 \\
\hline $\mathrm{T}_{4}$ & 6 & 5 \\
\hline \multicolumn{3}{|l|}{ Grading } \\
\hline $\mathrm{G}_{1}$ & 0 & 0 \\
\hline $\mathrm{G}_{2}$ & 6 & 2 \\
\hline $\mathrm{G}_{3}$ & 8 & 4 \\
\hline
\end{tabular}

$\mathrm{T}_{1}=\leq 2 \mathrm{~cm} ; \mathrm{T}_{2}=>2 \mathrm{~cm} ; \mathrm{T}_{3}=>5 \mathrm{~cm} ; \mathrm{T}_{4}=$ Tumor mit Thorax wand- oder Hautinfiltration; $G_{1}=$ hoch differenziert; $G_{2}=$ mässig differenziert; $\mathrm{G}_{3}=$ niedrig differenziert.

a Wenn Östrogen- und/oder Progesteronrezeptor positiv.

abreicht. In der metastasierten Situation ergab sich im Gesamtkollektiv eine Remissionsrate von 37\% (16\% komplette Remissionen). Nach $\mathrm{FE}_{50} \mathrm{C}$ betrug die Remissionsrate $34 \%$ (15\% komplete Remissionen) und nach $\mathrm{FE}_{75} \mathrm{C} 44 \%$ (19\% komplete Remissionen). Die mittlere Remissionsdauer betrug nach $\mathrm{FE}_{50} \mathrm{C}$ und $\mathrm{FE}_{75} \mathrm{C} 20,5$ bzw. 25 Wochen $(p=0,6)$. Die mittlere Überlebenszeit betrug nach $\mathrm{FE}_{50} \mathrm{C}$ und $\mathrm{FE}_{75} \mathrm{C} 60$ bzw. 85 Wochen $(\mathrm{p}=0,15$; Abb. 2). Die hämatologischen Toxizitäten wurden jeweils für $\mathrm{FE}_{50} \mathrm{C}$ oder $\mathrm{FE}_{75} \mathrm{C}$ für adjuvant behandelte Patientinnen und Patientinnen mit fortgeschrittenen Mammakarzinomen gemeinsam ausgewertet. Für $\mathrm{FE}_{50} \mathrm{C}$ lagen 77 und für $\mathrm{FE}_{75} \mathrm{C} 73$ auswertbare Zyklen vor. Im Standardtherapiearm lag der Leukozyten-Nadir am 8 . Tag bei 2,75 $\mathrm{G} / 1$ und nach dosisintensivierter Therapie am 14 . Tag bei 2,5 G/1. Der Thrombozyten-Nadir lag nach $\mathrm{FE}_{50} \mathrm{C}$ am 10. Tag bei $225 \mathrm{G} / 1$ und nach $\mathrm{FE}_{75} \mathrm{C}$ am 12 . Tag bei $220 \mathrm{G} / \mathrm{l}$. Im Standardtherapiearm lag der niedrigste Hämoglobinwert während der gesamten Therapie bei $10,75 \mathrm{~g} / \mathrm{dl}$ und nach dosisintensivierter Therapie bei $10,7 \mathrm{~g} / \mathrm{dl}$. Statistisch signifikante Unterschiede zwischen beiden Studiengrup-
Tabelle 4. Charakteristika von Patientinnen mit metastasierter Erkrankung

\begin{tabular}{|c|c|c|c|c|}
\hline & \multicolumn{2}{|c|}{$\mathrm{FE}_{75} \mathrm{C}$} & \multicolumn{2}{|c|}{$\mathrm{FE}_{50} \mathrm{C}$} \\
\hline & $\mathrm{n}$ & $\%$ & $\mathrm{n}$ & $\%$ \\
\hline Auswertbare Patientinnen & 16 & 100 & 27 & 100 \\
\hline Mittleres Alter, Jahre (Bereich) & 48 & $(30-72)$ & 47 & $(27-73)$ \\
\hline \multicolumn{5}{|l|}{ Hormonrezeptorstatus } \\
\hline Positiva $^{\mathrm{a}}$ & 9 & 56 & 16 & 59 \\
\hline Negativ & 7 & 44 & 11 & 41 \\
\hline Prämenopausal & 7 & 44 & 17 & 63 \\
\hline Postmenopausal & 9 & 56 & 10 & 37 \\
\hline \multicolumn{5}{|l|}{ Vorausgegangene Chemotherapie } \\
\hline Keine & 12 & 75 & 15 & 56 \\
\hline Adjuvant & 3 & 19 & 9 & 33 \\
\hline Metastasiert & 1 & 6 & 3 & 11 \\
\hline \multicolumn{5}{|l|}{ Anzahl befallene Organe ${ }^{b}$} \\
\hline 1 & 1 & 6 & 1 & 4 \\
\hline 2 & 8 & 50 & 8 & 30 \\
\hline 3 & 3 & 19 & 12 & 44 \\
\hline 4 & 4 & 25 & 6 & 22 \\
\hline
\end{tabular}

Es ergaben sich keine statistisch signifikanten Unterschiede in der Verteilung der Merkmale zwischen beiden Therapiegruppen (Chi-Square).

a Wenn Östrogen- und/oder Progesteronrezeptor positiv.

b Hepatisch, pulmonal/pleural, kutan, ossär, Zentralnervensystem oder andere.

pen bezüglich der hämatologischen Toxizitäten liessen sich nicht nachweisen. Die mittleren Leukozyten- und Hämoglobinwerte für $77 \mathrm{FE}_{50} \mathrm{C}$ - und für $73 \mathrm{FE}_{75} \mathrm{C}$-Zyklen sind in der Abbildung 3 dargestellt.

Eine Grad-III-Emesis trat nach $\mathrm{FE}_{50} \mathrm{C}$ und nach $\mathrm{FE}_{75} \mathrm{C}$ bei 33 bzw. $43 \%$ der Patientinnen auf. $\mathrm{Zu}$ einer Grad-IIIInfektion kam es bei 2 Patientinnen nach $\mathrm{FE}_{50} \mathrm{C}$. Epirubicin führte bei allen Patientinnen zu einer vollständigen, aber reversiblen Alopezie (Grad III). Unter $\mathrm{FE}_{50} \mathrm{C}$ oder $\mathrm{FE}_{75} \mathrm{C}$ wurden keine klinisch relevanten Kardiotoxizitäten (Grad III und IV) beobachtet. Die Dosisintensivierung von Epirubicin führte nicht zu einer Erhöhung von klinisch relevanten Toxizitäten (WHO-Grad III und IV).

\section{Diskussion}

In unserem adjuvant therapierten Kollektiv waren rezidivfreies Überleben und Gesamtüberleben nach 4 Jahren mit 30 bzw. $45 \%$ deutlich niedriger als in vergleichbaren Studien über den Einsatz von Anthrazyklinen in der adjuvanten Therapie (Tab. 1). Die in der Tabelle 3 dargestellten Patientencharakteristika verdeutlichen, dass Patientinnen dieses Kollektives ein deutlich erhöhtes Rezidivrisiko aufwiesen. So waren im Mittel neun axilläre 

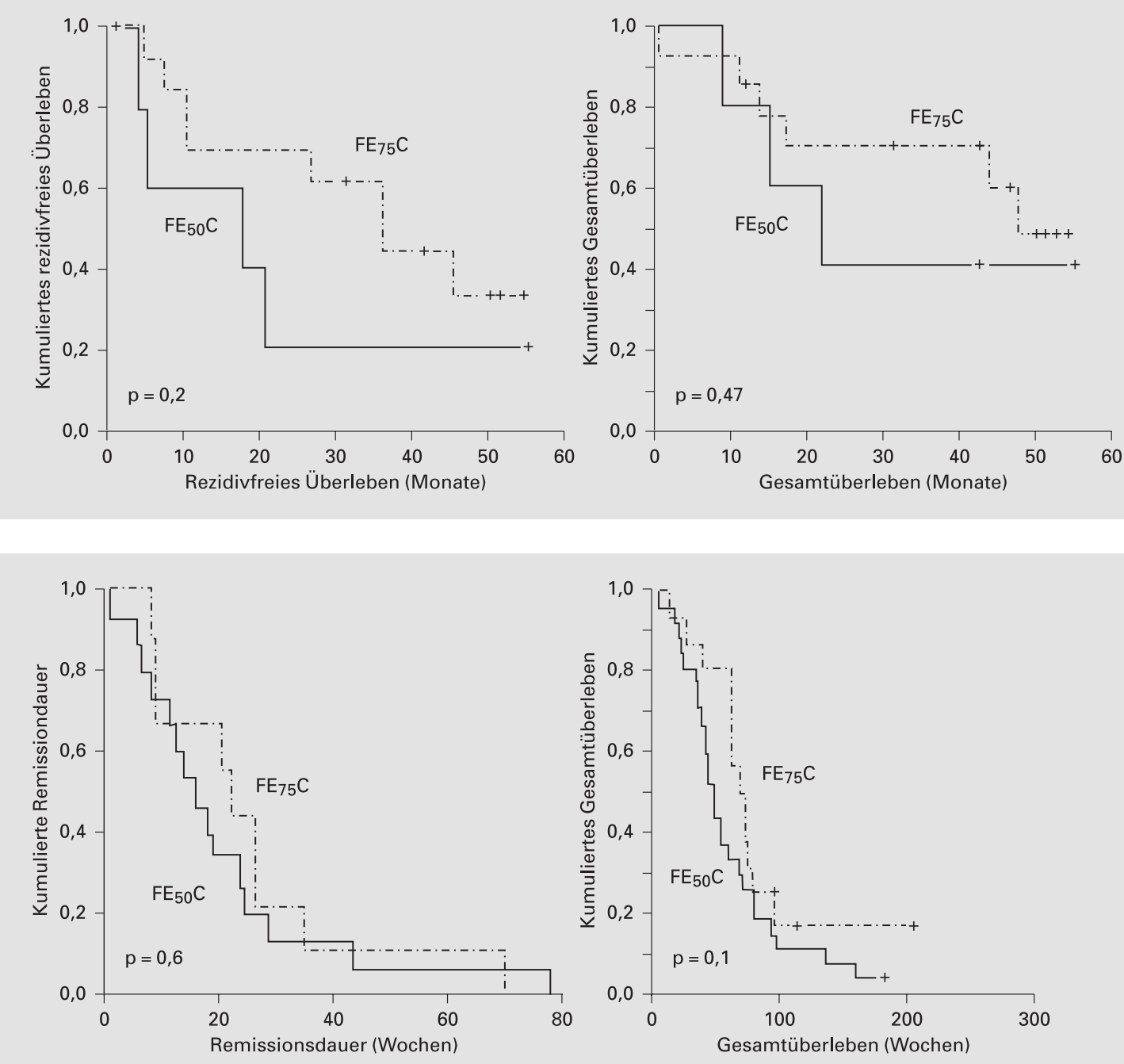

Abb. 1. Rezidivfreies und Gesamtüberleben nach Kaplan und Meier [22] nach adjuvanter Chemotherapie mit $\mathrm{FE}_{50} \mathrm{C}$ und $\mathrm{FE}_{75} \mathrm{C}$.

Abb. 2. Remissionsdauer und Gesamtüberleben nach Kaplan und Meier [22] von metastasierten Patientinnen nach $\mathrm{FE}_{50} \mathrm{C}$ - und $\mathrm{FE}_{75} \mathrm{C}$-Therapie.

Abb. 3. Verläufe der Leukozyten- und Hämoglobinwerte im Mittel mit Standardabweichungen für jeweils 77 und 73 auswertbare $\mathrm{FE}_{50} \mathrm{C}$ - bzw. $\mathrm{FE}_{75} \mathrm{C}$-Zyklen.
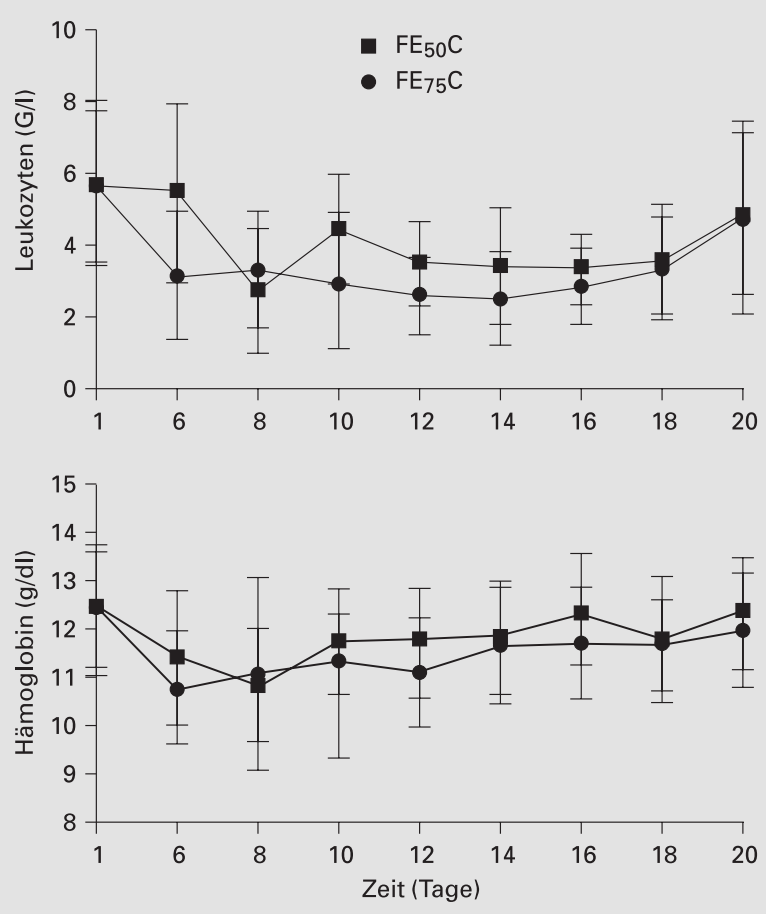
Tabelle 5. Randomisierte Studien, in denen eine Dosis-Wirkungs-Beziehung von Anthrazyklinen in der adjuvanten Therapie des nodalpositiven Mammakarzinoms untersucht wurde

\begin{tabular}{|c|c|c|c|c|c|c|c|}
\hline Regime & $\begin{array}{l}\text { Dosierung } \\
\mathrm{mg} / \mathrm{m}^{2}\end{array}$ & $\mathrm{n}$ & $\begin{array}{l}\mathrm{DI} \mathrm{mg} / \mathrm{m}^{2} / \\
\text { Woche }\end{array}$ & $\begin{array}{l}\text { Follow-up- } \\
\text { Periode } \\
\text { Monate }\end{array}$ & $\begin{array}{l}\text { Rezidivfreies } \\
\text { Überleben } \\
\%\end{array}$ & $\begin{array}{l}\text { Gesamt- } \\
\text { überleben } \\
\%\end{array}$ & Literatur \\
\hline CAF & $\begin{array}{l}300 / 30 / 300 q 4 w \\
600 / 60 / 600 q 4 w\end{array}$ & 1572 & $\begin{array}{l}7.5 \\
15\end{array}$ & 36 & $\begin{array}{l}65 \\
75^{*}\end{array}$ & - & 17 \\
\hline $\mathrm{A} / \mathrm{CMF}$ & $\begin{array}{l}\text { alternierende Gabe } \\
\text { sequentielle Gabe }\end{array}$ & 403 & $\begin{array}{r}9 \\
25\end{array}$ & 120 & $\begin{array}{l}28 \\
42^{*}\end{array}$ & $\begin{array}{l}44 \\
58^{*}\end{array}$ & 25 \\
\hline FEC & $\begin{array}{l}500 / 50 / 500 \mathrm{q} 3 \mathrm{w} \\
500 / 100 / 500 \mathrm{q} 3 \mathrm{w}\end{array}$ & 565 & $\begin{array}{l}16 \\
30\end{array}$ & 60 & & $\begin{array}{l}70 \\
80 *\end{array}$ & 16 \\
\hline $\begin{array}{l}\mathrm{EC} / \mathrm{CMF} \\
\mathrm{EC}\end{array}$ & $\begin{array}{l}90 / 600 \mathrm{q} 3 \mathrm{w} \\
120 / 600 \mathrm{q} 2 \mathrm{w}\end{array}$ & 180 & $\begin{array}{l}29 \\
54\end{array}$ & 26 & $\begin{array}{l}43 \\
65^{*}\end{array}$ & & 24 \\
\hline $\begin{array}{l}\mathrm{CMF} \\
\mathrm{FEC}\end{array}$ & $\begin{array}{l}1400 / 80 / 1200 \mathrm{q} 4 \mathrm{w} \\
1000 / 120 / 1050 \mathrm{q} 4 \mathrm{w}\end{array}$ & 710 & 30 & 60 & $\begin{array}{l}53 \\
63^{*}\end{array}$ & $\begin{array}{l}70 \\
77^{*}\end{array}$ & 7 \\
\hline
\end{tabular}

$\mathrm{C}=$ Cyclophosphamid; $\mathrm{A}=$ Doxorubicin; $\mathrm{F}=$ 5-Fluorouracil; $\mathrm{M}=$ Methotrexat $\mathrm{E}=$ Epirubicin; $\mathrm{q} 4 \mathrm{w}$ = 4-wöchentlich; q3w = 3-wöchentlich; q2w = 2-wöchentlich.

* Signifikanter Unterschied.

Lymphknoten befallen, 50\% der Tumoren waren hormonrezeptornegativ, und 14 von 20 Primärtumoren waren $>5 \mathrm{~cm}$ bzw. hatten eine Haut- oder Pektoralisinfiltration zur Folge. Alle Primärtumoren waren schlecht oder mässig differenziert. Ein inflammatorisches Mammakarzinom wiesen 6 von 20 Patientinnen auf. Während Anthrazykline für die Therapie des metastasierten Mammakarzinoms eine gesicherte Indikation haben, sind die potentiellen Vorteile in der adjuvanten Therapie bisher weniger gut belegt. In der NSABP B-11/12-Studie [2] wurde bei lymphknotenpositiven, hormonrezeptornegativen Patientinnen ein signifikanter Vorteil bezüglich rezidivfreiem Überleben und Gesamtüberleben im mit Anthrazyklin behandelten Therapierarm beobachtet. Bemerkenswert ist, dass in einer aktuellen Auswertung dieser Studie der Vorteil einer anthrazyklinhaltigen Therapie insbesondere bei ErbB-2-positiven Patientinnen ausgeprägt war [23]. Ebenso konnten in einer französischen Studie von Misset et al. [1] rezidivfreies Überleben und Gesamtüberleben nach 10 Jahren durch eine anthrazyklinhaltige Therapie signifikant verbessert werden. In einer aktuellen schwedischen Studie von Mouridsen et al. [6] führte FEC beim prämenopausalen Mammakarzinom - Einschlusskriterien: nodal negativ $\left(\mathrm{G}_{2}-\mathrm{G}_{3}\right)$ oder nodal positiv, Hormonrezeptor negativ nach 6 Jahren im Vergleich zu CMF zu einer signifikanten Verbesserung des Gesamtüberlebens $(p=0.01)$. In der NSABP B-15-Studie [5] konnte dagegen nach einer 3-jährigen Beobachtungszeit bei lymphknotenpositiven, rezeptornegativen Patientinnen bisher keine signifikante Verbesserung des rezidivfreien oder des Gesamtüberlebens durch eine anthrazyklinhaltige Therapie mit Doxorubicin im Vergleich zu CMF erzielt werden. Levine et al. [7] konnten in einer kanadischen Studie wiederum durch ein intensiviertes FEC-Schema im Vergleich zu CMF bei prämenopausalen, lymphknotenpositiven Patientinnen einen signifikanten Vorteil in bezug auf rezidivfreies und Gesamtüberleben erreichen. Vor dem Hintergrund dieser Studien scheint der Einsatz von Anthrazyklinen in der adjuvanten Therapie, insbesondere bei einem erhöhten Rezidivrisiko, gerechtfertigt zu sein. Unklar ist jedoch weiterhin der Stellenwert einer Dosisintensivierung von Anthrazyklinen in der adjuvanten Therapie des Mammakarzinoms. In unserer Untersuchung hatten Patientinnen nach dosisintensivierter Therapie längere rezidivfreie Intervalle und höhere Gesamtüberlebensraten. Diese Unterschiede waren jedoch statistisch nicht signifikant, was möglicherweise auf die geringe Zahl der untersuchten Patientinnen zurückzuführen ist. Das es sich um eine retrospektive Untersuchung handelt, in welcher die Ergebnisse durch die Selektion und die ungleiche Verteilung von Patientinnen mit unterschiedlicher Prognose in den jeweiligen Behandlungsarmen erheblich beinflusst werden können, sind allgemeine Schlussfolgerungen nicht zulässig. Die Ergebnisse retrospektiver Untersuchungen können aber die Grundlage für eine Entwicklung neuer Behandlungskonzepte sein, die in prospektiven Studien weiter überprüft werden müssen. Unsere Ergebnisse bestätigen die Notwendigkeit, eine Dosisintensivierung mit Anthrazyklinen beim Mammakarzinom in weiteren prospektiven Studien zu untersuchen. Bisher sind nur drei prospektiv randomisierte Studien, die in der adjuvanten Therapie des Mammakarzinoms eine echte Dosisintensivierung von Anthrazyklinen untersucht haben, veröffentlich worden [7, 16, 24], und nur zwei dieser Studien [16, 24] setzten Anthrazykline in beiden Studien- 
armen ein (Tab. 5). Die Studie der French Adjuvant Study Group [16] (Einschlusskriterien: $>3$ positive axilläre Lymphknoten oder 1-3 Lymphknoten und negativer Hormonrezeptorstatus und hohes Grading) konnte durch die Verdoppelung der Epirubicin-DI eine signifikante Verbesserung der Gesamtüberlebensrate nachweisen. Gemeinsam mit der Frauenklinik Rechts der Isar der Technischen Universität München, der Universitätsfrauenklinik Hamburg-Eppendorf und dem Klinikum Landshut haben wir im Rahmen einer randomisierten Studie eine Dosisintensivierung von Epirubicin bei 180 Mammakarzinompatientinnen mit mehr als neun positiven axillären Lymphknoten oder mit perinodaler Lymphknoteninfiltration untersucht [24]. Die Patientinnen erhielten entweder Epirubicin/Cyclophosphamid 120/600 mg/m² 14-tägig mit Gabe von G-CSF (Filgrastim) von Tag 2 bis Tag 12 oder eine EC/CMF-Therapie in Standarddosierung. Durch die Dosisintensivierung konnte eine signifikante Verlängerung des rezidivfreien Intervalls bereits nach einer kurzen Beobachtungszeit von 26 Monaten erzielt werden.

In einer amerikanischen Studie der Cancer and Leukemia Group B [17] führte die Dosisintensivierung eines FAC-Regimes beim lymphknotenpositiven Mammakarzinom zu einer signifikanten Verbesserung des rezidivfreien Überlebens. Ebenso führte die Erhöhung der DI durch eine sequentielle Applikation von Doxorubicin in der Studie von Bonadonna et al. [25] zu einem verbesserten Therapieansprechen. Beide Studien werden häufig zitiert, um den Benefit einer Dosisintensivierung in der adjuvanten Therapie des Mammakarzinoms zu belegen. Der signifikante Unterschied in diesen Studien ist jedoch am ehesten auf das Unterschreiten einer erforderlichen Schwellendosis in den sehr niedrig dosierten Vergleichsarmen zurückzuführen.

Beim metastasierten Mammakarzinom ist durch Studien gut belegt, dass anthrazyklinhaltige Regime zu höheren Remissionsraten, längeren Remissionsdauern und teilweise zu einer Verbesserung des Gesamtüberlebens führen können [26]. Der Nutzen einer Dosisintensivierung von Anthrazyklinen beim metastasierten Mammakarzinom scheint dagegen geringer zu sein, da in bisherigen Studien nur eine Verbesserung der Remissionsraten und der Remissionsdauer, aber keine Verbesserung des Gesamtüberlebens erzielt werden konnte (Tab. 6) [18-21, 27, 28]. In unserer Untersuchung liess sich die Remissionsrate durch eine Dosisintensivierung von 34 auf 44\% erhöhen. Die mittlere Remissionsdauer und das Gesamtüberleben unterschieden sich zwischen beiden Kollektiven jedoch nicht signifikant. Da eine Erhöhung der DI insbesondere bei der metastasierten Erkrankung zu höheren Toxizitäten und damit zu einer grösseren Belastung für die Patientin führen kann, ist eine Dosisintensivierung von Anthrazyklinen trotz höherer Remissionsraten
Tabelle 6. Randomisierte Studien, in denen eine Dosis-WirkungsBeziehung von anthrazyklinhaltigen Kombinationschemotherapien oder Monochemotherapien in der metastasierten Situation untersucht wurde

\begin{tabular}{llllll}
\hline Regime & $\begin{array}{l}\text { Dosierung } \\
\mathrm{mg} / \mathrm{m}^{2}\end{array}$ & $\mathrm{n}$ & \multicolumn{2}{l}{$\begin{array}{l}\text { Remissions- Gesamt- } \\
\text { rate, } \%\end{array}$} & $\begin{array}{l}\text { Literatur } \\
\text { überleben } \\
\text { Monate }\end{array}$ \\
\hline FEC & $500 / 50 / 500$ & 456 & 41 & 17 & 19 \\
& $500 / 100 / 500$ & & $57^{*}$ & 18 & \\
\hline FEC & standard & 62 & 42 & 17 & 27 \\
& intensiviert & & 69 & 24 & \\
\hline FAC & standard & 59 & 78 & 20 & 28 \\
& intensiviert & & 78 & 20 & \\
\hline EP & 50 & 211 & 23 & 11 & 21 \\
& 100 & & $41^{*}$ & 10 & \\
\hline A & 35 & 48 & 25 & 8 & 20 \\
& 70 & & $58^{*}$ & 20 & \\
\hline E & 40 & 287 & 20 & 14 & 18 \\
& 60 & & 20 & 14 & \\
& 90 & & $38^{*}$ & 15 & \\
& 135 & & 36 & 11 & \\
\hline
\end{tabular}

$\mathrm{F}=$ 5-Fluorouracil; $\mathrm{E}=$ Epirubicin; $\mathrm{C}=$ Cyclophosphamid, $\mathrm{A}=$ Doxorubicin; $\mathrm{P}=$ Prednisolon.

* Signifikanter Unterschied.

bei unverändertem Gesamtüberleben beim metastasierten Mammakarzinom ausserhalb von Studien nicht gerechtfertigt. Die Einhaltung der geplanten DI scheint dagegen jedoch wichtig zu sein, da das Unterschreiten einer erforderlichen Schwellendosis die Effektivität deutlich senken kann. Da Symptomlinderung und eine Verbesserung der Lebensqualität Therapieziele darstellen, kann in selten Fällen eine dosisintensivierte anthrazyklinhaltige Therapie indiziert sein, um rascher und zuverlässiger Beschwerden lindern zu können. Die möglichen Therapievorteile müssen im Einzelfall gegenüber den zu erwartenden Nebenwirkungen abgewogen werden.

Zusammenfassend rechtfertigen unsere Ergebnisse, die in Einklang mit den Ergebnissen prospektiver Studien stehe, den Einsatz von Anthrazyklinen beim metastasierten Mammakarzinom oder in der adjuvanten Therapie bei erhöhtem Rezidivrisiko. Die Einhaltung der geplanten DI ist auf Grund der beobachteten Dosis-Wirkungs-Beziehung von Anthrazyklinen zur Optimierung des Therapieerfolges notwendig. Empfehlungen zur Dosisintensivierung von Anthrazyklinen ausserhalb von Studien können derzeit aber noch nicht gegeben werden.

\section{Dank}

Die vorliegende Auswertung wurde ermöglicht durch die Unterstützung der Ernst und Berta Grimmke-Stiftung, Düsseldorf. 


\section{Literatur}

1 Misset JL, di Palma M, Dalgado M, Plagne R, Chollet PH, Fumoleau P, Le Mevel B, Belpomme D, Guerrin J, Fargeot P, Metz R, Ithzaki M, Hill C, Mathe G: Adjuvant treatment of node-positive breast cancer with cyclophosphamide, doxorubicin, fluorouracil, and vincristine versus cyclophosphamide, methotrexate, and fluorouracil: Final results after a 16-year median follow-up duration. J Clin Oncol 1996; 14:1136-1145.

2 Fisher B, Redmond C, Wickerham DL, Bowman D, Schipper H, Wolmark N, Sass R, Fisher ER, Jochimsen P, Legault-Poisson S: Doxorubicin-containing regimens for the treatment of stage II breast cancer: NSABP B-11/12. J Clin Oncol 1989;7:572-582.

3 Coombes RC, Bliss JM, Wils J, Morvan F, Espie M, Amadori D, Gambrosier P, Richards M, Aapro M, Villar-Grimalt A, McArdle C, Perez-Lopez FR, Vassilopoulos P, Ferreira EP, Chilvers CE, Coombes G, Woods EM, Marty M: Adjuvant cyclophosphamide methrotrexate, and fluorouracil versus fluorouracil, epirubicin, and cyclophosphamide chemotherapy in premenopausal women with axillary node-positive operable breast cancer: Results of a randomized trial. The International Collaborative Cancer Group. J Clin Oncol 1996;14:35-45.

4 Budd GT, Green S, O'Bryan RM, Martino S, Abeloff MD, Rinehart JJ, Hahn R, Harris J, Tormey D, O’Sullivan J: Short course FAC-M vs. 1 year of CMFVP in node-positive hormone receptor-negative breast cancer: An intergroup study. J Clin Oncol 1995; 13:831.

5 Fisher B, Brown AM, Dimitrov NV, Poisson R, Redmond C, Margolese RG, Bowman D, Wolmark N, Wickerham DL, Kardinal CG: Two months of doxorubicin-cyclophosphamide with and without intervall reinduction therapy compared with 6 months of cyclophosphamide, methotrexate, and fluorouracil in positive-node breast cancer patients with tamoxifen-nonresponsive tumors: Results from the National Surgical Adjuvant Breast and Bowel Project B-15. J Clin Oncol 1990;8:1483-1496.

6 Mouridsen HAT, Andersen J, Andersson M, Dombernowsky P, Ejlertsen B, Rose C, Sørensen S, Sandberg E, Andersen KW, Jensen MB, Bengtson NO, Bergh J, Nordenskjøld B, Swedish Oncology Centers Umeå, Uppsala, Linköping, Sweden: Adjuvant anthraycycline in breast cancer: Improved outcome in premenopausal patients following substitution of methotrexate in CMF with epirubicin (abstract). Proc Am Soc Clin Oncol 1999; 18:254.

7 Levine M, Bramwell VH, Pritchard KI, Norris BD, Shepherd LE, Abu-Zahra H, Findlay B, Warr D, Bowman D, Myles J, Arnold A, Vandenberg T, MacKenzie R, Robert J, Ottaway J, Burnell M, Williams CK, Tu D: Randomized trial of intensive cylophosphamide, epirubicin, and fluorouracil chemotherapy compared with cyclophosphamide, methotrexate, and fluorouracil in premenopausal women with nodepositive breast cancer. National Cancer Institute of Canada Clinical Trials Group. J Clin Oncol 1998;16:2651-2658.
8 Carpenter JT, Velez-Garcia E, Arton BS: Five year results of a randomized comparison of cyclophosphamide, doxorubicin and fluorouracil (CAF) vs. cyclophosphamide, methotrexate and fluorouracil (CMF) for node-positive breast cancer: Southeastern Cancer Study Group (abstract). Proc Am Soc Clin Oncol 1993; 13:68.

9 Tormey DC, Weinberg VE, Leone LA, Gildewell OJ, Perloff M, Kennedy BJ, Cortes E, Silver RT, Weiss RB, Aisner J: A comparison of intermittent vs. continuous and of Adriamycin vs. methotrexate 5-drug chemotherapy for advanced breast cancer. A Cancer and Leukemia Group B study. Am J Clin Oncol 1984;7:231239.

10 Aisner J, Weinberg V, Perloff M, Weiss R, Perry M, Korzun A, Ginsberg S, Holland JF: Chemotheapy vs. chemoimmunotherapy for metastatic carcinoma of the breast: A CALGB study. Cancer and Leukemia Group B. J Clin Oncol 1987;5:1523-1533.

11 Bull JM, Tormey DC, Li SH, Carbone PP Falkson G, Blom J, Perlin E, Simon R: A randomised comparative trial of Adriamycin versus methotrexate in combination drug therapy. Cancer 1978;41:1649-1657.

12 Cummings FJ, Gelman R, Horton J: Comparison of CAF versus CMFP in metastatic breast cancer: Analysis of prognostic factors. J Clin Oncol 1985;3:932-940.

13 Smalley RV, Lefante J, Bartolucci A, Carpenter J, Vogel C, Krauss S: A comparison of cyclophosphamide, Adriamycin, and 5-Fluorouracil (CAF) and cyclophosphamide, methotrexate, 5-Fluourouracil, vincristine, and prednisone (CMFVP) in patients with advanced breast cancer. Breast Cancer Res Treat 1983;3:209220.

14 Bonadonna G, Valagussa P. Dose-response effect of adjuvant chemotherapy in breast cancer. N Engl J Med 1981;304:10-15.

15 Hryniuk W, Frei E 3rd, Wright FA: A single scale for comparing dose-intensity of all chemotherapy regimens in breast cancer: Summation dose-intensity. J Clin Oncol 1998;16: 3137-3147.

16 Bonneterre J, Roche H, Bremond A, French Adjuvant Study Group: A randomized trial of adjuvant chemotherapy with FEC 50 versus FEC 100 for node-positive operable breast cancer: Early report (abstract). Proc Am Soc Clin Oncol 1996;15:104.

17 Wood WC, Budmann DR, Korzun AH, Cooper MR, Younger J, Hart RD, Moore A, Ellerton JA, Norton L, Ferree CR: Dose and dose intensity of adjuvant chemotherapy for stage II node-positive breast carcinoma. N Engl J Med 1994;330:1253-1259.

18 Bastholt L, Dalmark M, Gjedde SB, Pfeiffer P, Pedersen D, Sandberg E, Kjær M, Mouridsen HT, Rose C, Nielsen OS, Jakobsen P, Benzen SM: Dose-response relationship of epirubicin in the treatment of postmenopausal patients with metastatic breast cancer: A randomized study of epirubicin at four different dose levels performed by the Danish Breast Cancer Cooperative Group. J Clin Oncol 1996;14:11461155 .
19 Brufman G, Colajori E, Ghilezan N, Lassus M, Martoni A, Perevodchikova N, Tosello C, Viaro D, Zielinski C: Doubling epuribicin dose intensity $\left(100 \mathrm{mg} / \mathrm{m}^{2}\right.$ versus $\left.50 \mathrm{mg} / \mathrm{m}^{2}\right)$ in the FEC regimen significantly increases response rates: An international randomised phase III study in metastatic breast cancer. The Epirubicin High Dose (HEPI 010) Study Group. Ann Oncol 1997;8:155-162.

20 Carmo-Pereira J, Costa FO, Henriques E, Godinho F, Cantino-Lopes MG, Sales-Luis A, Rubens RD: A comparison of two doses of Adriamycin in the primary chemotherapy of disseminated breast carcinoma. Br J Cancer 1987;56: 471-473.

21 Habeshaw T, Paul J, Jones R, Stallard S, Stewart M, Kaye SB, Soukop M, Symonds RP, Reed NS, Rankin EM: Epirubicin at two dose levels with prednisolone as treatment for advanced breast cancer: The results of a randomized trial. J Clin Oncol 1991;9:295-304.

22 Kaplan EL, Meier P: Nonparametric estimation from incomplete observations. J Am Stat Assoc 1958;53:457-481.

23 Paik S, Bryant J, Park C, Fisher B, Tan-Chiu E, Hyams D, Fisher ER, Lippman ME, Wickerham DL, Wolmark N: erbB-2 and response to doxorubicin in patients with axillary lymph node-positive, hormone receptor-negative breast cancer. J Natl Cancer Inst 1998;90: 1361-1370.

24 Thomssen C, Untch M, Schill E, Sattler D, Konecny G, Oberlechner D, Hoffmann R, Kahlert S, Kuhn W, Ulm K, Hepp H, Graeff H, Jänicke F: Dose-intensive adjuvant chemotherapy of high-risk breast cancer patients. First results of a prospective randomized study. J Cancer Res Clin Oncol 1997;124(suppl): P1.0926.

25 Bonadonna G, Zambetti M, Valagussa P: Sequential or alternating doxorubicin and $\mathrm{CMF}$ regimes in breast cancer with more than three positive nodes: Ten-year results. JAMA 1995; 273:542-547.

26 A'Hern RP, Smith IE, Ebbs SR: Chemotherapy and survival in advanced breast cancer: The inclusion of doxorubicin in Cooper type regimens. Br J Cancer 1993;67:801-805.

27 Ardizzoni A, Venturini M, Sertoli MR, Giannessi PG, Brema F, Danova M, Testore F, Mariani GL, Pennucci MC, Queriolo P: Granulocyte-macrophage colony-stimulating factor allows acceleration and dose intensity increase of CEF chemotherapy: A randomized study in patients with advanced breast cancer. Br J Cancer 1994;69:385-391.

28 Hortobagyi GN, Bodey GP, Buzdar AU, Frye D, Legah SS, Malik R, Smith TL, Blumenschein GR, Yap HY, Rodriquez V: Evaluation of high-dose versus standard FAC chemotherapy for advanced breast cancer in protected environment units: A prospective randomized study. J Clin Oncol 1987;5:354-364. 